\title{
Criminologie
}

\section{Caractéristiques et évolution du terrorisme à motif religieux au Canada depuis 1973}

\section{Stéphane Leman-Langlois}

Volume 41, numéro 2, automne-hiver 2008

Les organisations dites sectes, les lois et la société

URI : https://id.erudit.org/iderudit/019431ar

DOI : https://doi.org/10.7202/019431ar

Aller au sommaire du numéro

\section{Éditeur(s)}

Les Presses de l'Université de Montréal

ISSN

0316-0041 (imprimé)

1492-1367 (numérique)

Découvrir la revue

Citer cet article

Leman-Langlois, S. (2008). Caractéristiques et évolution du terrorisme à motif religieux au Canada depuis 1973. Criminologie, 41(2), 9-29.

https://doi.org/10.7202/019431ar
Résumé de l'article

Depuis le milieu des années 1990, plusieurs auteurs ont cru reconnaître l'apparition de ce qu'ils ont appelé un " nouveau terrorisme ». C'est un terrorisme dont les cibles et les sources dépassent le cadre des États, aux objectifs glorieux et à fort contenu surnaturel ou légendaire - faisant donc peu de revendications concrètes ou réalisables. C'est aussi, voire surtout, un terrorisme à fort contenu religieux, absolutiste et accompagné d'une violence qui ne connaît plus les limites établies par le terrorisme conventionnel. Pour des raisons à la fois théoriques et pratiques, il est important de comprendre comment et à quel point ce nouveau terrorisme se manifeste au Canada. À partir d'une banque de données qualitatives de près de 400 incidents et situations de violence politique au Canada depuis 1973, cet article établit certains faits, dont la rareté des attentats religieux et leur presque disparition depuis les années 1980. Cette évolution est ensuite analysée et quelques explications possibles sont offertes, dont la fonction de contrôle social souvent attribuée à la pratique religieuse. En conclusion, nous révisons la pertinence du concept de « nouveau terrorisme » et son utilité à la fois théorique et pratique. 


\title{
Caractéristiques et évolution du terrorisme à motif religieux au Canada depuis 1973
}

\author{
Stéphane Leman-Langlois \\ Centre international de criminologie comparée \\ s.langlois@umontreal.ca
}

RÉSUMÉ - Depuis le milieu des années 1990, plusieurs auteurs ont cru reconnaître l'apparition de ce qu'ils ont appelé un «nouveau terrorisme». C'est un terrorisme dont les cibles et les sources dépassent le cadre des États, aux objectifs glorieux et à fort contenu surnaturel ou légendaire - faisant donc peu de revendications concrètes ou réalisables. C'est aussi, voire surtout, un terrorisme à fort contenu religieux, absolutiste et accompagné d'une violence qui ne connaît plus les limites établies par le terrorisme conventionnel. Pour des raisons à la fois théoriques et pratiques, il est important de comprendre comment et à quel point ce nouveau terrorisme se manifeste au Canada.

À partir d'une banque de données qualitatives de près de 400 incidents et situations de violence politique au Canada depuis 1973, cet article établit certains faits, dont la rareté des attentats religieux et leur presque disparition depuis les années 1980. Cette évolution est ensuite analysée et quelques explications possibles sont offertes, dont la fonction de contrôle social souvent attribuée à la pratique religieuse. En conclusion, nous révisons la pertinence du concept de «nouveau terrorisme» et son utilité à la fois théorique et pratique.

\section{Introduction}

En criminologie et en sociologie, la religion et la pratique religieuse ont surtout été conçues et examinées en tant que facteurs de socialisation, de contrôle social ou de normalisation. Dans tous les cas, il s'agissait de religions et sectes chrétiennes et en particulier de groupes protestants, dont on a mesuré l'effet anticriminogène, avec des résultats mitigés (Hirschi et Stark, 1969; Johnson et al., 2001; Heaton, 2006). Dans la plupart des cas, ces études ont pris place dans des sociétés 
relativement homogènes et la religion observée était clairement dominante socialement, culturellement et politiquement. Certaines études, dont celle de Gusfield (1986) ont porté sur les conflits politiques surgissant entre les groupes socioreligieux. Dans son étude sur la prohibition, Gusfield a bien montré comment la moralité religieuse fut mobilisée pour maintenir la domination politique de la classe anglosaxonne protestante aux États-Unis. Bref, qu'elle soit une force de socialisation bénéfique ou d'oppression politique, la religion reste conçue comme un élément de contrôle, de normalisation.

À la fois, depuis quelques années, une littérature importante s'est accumulée autour de la question des activités de diverses «sectes» (parfois désignées sous l'euphémisme souvent incorrect de «nouvelles religions»). Dans l'ensemble, cette littérature s'est concentrée sur certains événements particulièrement spectaculaires, comme le meurtre/ suicide des membres de la secte de Jim Jones en Guyane (Chidester, 1988; Hall, 1995), le suicide collectif des initiés du groupe Heaven's Gate (Davis, 2000; Urban, 2000), l'implosion toujours nébuleuse de l'Ordre du Temple Solaire (Introvigne, 1995; Introvigne et Mayer, 2002) et l'attentat au sarin de la secte Aum (Metraux, 1995; Campbell, 1999; Lifton, 2000). Bien sûr, la plupart des groupes sectaires ne commettent pas de telles violences, même si dans l'imaginaire populaire le mot «secte» est souvent juxtaposé à celui de «suicide» (Dein et Littlewood, 2000: 110). Plusieurs pays ont d'ailleurs choisi de surveiller de très près les activités des «sectes nuisibles», dont la Belgique et la France (avec la Mission interministérielle de vigilance et de lutte contre les dérives sectaires, www.miviludes.gouv.fr). Ces programmes sont explicitement justifiés par des références à des débordements de violence médiatisés. Dans ce discours, les «sectes», contrairement aux «religions», sont des facteurs de déviance, de violence antisociale.

L'évolution récente du phénomène du terrorisme a soulevé une question différente. Il s'agit d'actes de gravité extrême, justifiés par les préceptes religieux non pas d'une secte - groupe doté d'une dynamique assez particulière communément associée aux débordements de violence - mais bien d'une des plus importantes religions de la planète. Lapproche classique du «lavage de cerveau» ou "endoctrinement» (Baron, 2000) est donc peu appropriée puisque le groupe de référence n'est pas sectionné du reste de la société par une vision sectaire au sens habituel du terme. Bien sûr, on peut toujours imaginer que le groupe terroriste religieux se referme, devient l'équivalent d'une «secte» en 
adoptant sa propre version de la théologie dominante (pour un cas réel, voir Noble, 1998). Cependant, en général les groupes terroristes religieux, musulmans par exemple, agissent au nom d'une identité collective qu'ils croient victimisée par divers phénomènes ou entités indésirables (la mondialisation, l'impiété, la corruption, l'impérialisme, les «croisés», les «Juifs», les «Américains», George Bush, etc.). Plutôt que de s'isoler du monde, ils s'identifient comme ses plus dignes représentants. Élément plus important encore, la plupart des études empiriques sur le terrorisme ont rejeté l'hypothèse de l'endoctrinement religieux ou du «fanatisme» comme cause du passage à l'acte terroriste: même dans le cas du terrorisme suicide, sans doute le plus extrême, Pape (2005) et Sageman (2004) ont montré à quel point il est difficile de détecter une quelconque forme d'intoxication psychique chez le jihadiste moyen.

Ainsi, cette étude veut contribuer à la compréhension du terrorisme contemporain au Canada en se penchant sur le rôle qu'y tient la religion. Ceci impose en tout premier lieu d'évaluer l'importance relative du terrorisme à motif religieux à l'intérieur du phénomène du terrorisme en général, d'en identifier les différences et d'en comprendre le développement particulier, le choix des cibles et des méthodes.

Le terrorisme religieux contemporain a fait l'objet d'une distinction fondamentale chez plusieurs experts du terrorisme qui l'ont qualifié, prosaïquement, de «nouveau terrorisme» (Laqueur, 2000; Wilkinson, 2006). Cependant, un certain flou persiste toujours sur ce qui, au juste, est nouveau dans cette forme de terrorisme. Inutile de souligner qu'il ne s'agit pas de son aspect religieux en tant que tel: plusieurs phénomènes terroristes ayant marqué l'histoire (les Zélotes, les Assassins, les Thugs, etc.) avaient une base religieuse. Laqueur, entre autres, désigne plutôt la possibilité que des armes de destruction massive soient utilisées (chimiques, biologiques, radiologiques ou nucléaires, ou CBRN) comme nouvelle. Pourtant, dans ce cas, il y a peu à se mettre sous la dent puisque ce type d'attaque est d'une rareté extrême (on peut noter le sarin dans le métro de Tokyo, en mars 1995, et l'anthrax dans le courrier à Washington à l'automne 2001). D'autres auteurs (Stern, 1999; pour un regard plus sceptique, Sprinzak, 1998) soulignent plutôt la gravité sensiblement plus élevée des actes commis ou entrepris. On note par exemple que depuis les années 1980, le nombre de victimes d'attentats terroristes internationaux est en hausse, même si le nombre d'attentats est en baisse (Rand/MITP, 2007). La difficulté de cette approche, évidemment, réside dans l'identification d'un seuil de gravité 
objective au-delà duquel un acte terroriste doit être inclus dans la catégorie «nouveau terrorisme»: cet exercice nous rapproche de la tautologie, où l'existence d'une catégorie non vide de «nouveau terrorisme» dépend d'une définition à géométrie variable (Duyvesteyn, 2004).

Cela dit, la virtuelle disparition de l'Occident du terrorisme religieux, depuis l'époque des Lumières, lui donne effectivement un certain air de nouveauté relative. En fait, il est possible que le terrorisme religieux contemporain frappe surtout, justement, par sa contre-modernité, son apparence anachronique aux yeux occidentaux (Rappoport, 1990). Dans l'esprit de la modernité, les revendications politiques, juridiques, économiques et territoriales sont jugées rationnelles, compréhensibles, mais la violence fondée sur des croyances religieuses semble absurde. Cette facette caractéristique, sa justification par un absolu reposant sur des fondations surnaturelles, est un élément qui peut paraître secondaire (qu'importe la justification à ceux qui en meurent), mais qui porte à conséquence au plan théorique aussi bien qu'à celui de l'intervention. Si dans les années 1960, 1970 et 1980 «les terrorismes» (l'expression regroupe des cas de nature souvent radicalement différente) étaient surtout axés sur des revendications politiques relativement concrètes, le terrorisme à motif religieux ne comporte aucune revendication de ce genre. Il s'articule autour d'objectifs grandioses et justifie les tactiques immodérées censées les réaliser à l'aide de langages, de logiques, de préceptes et de concepts tirés d'une pensée religieuse.

Cela le distingue des autres formes de violence politique sous deux aspects fondamentaux. Premièrement, il repose sur un ensemble de référents qui renvoient à un univers surnaturel, c'est-à-dire dont les éléments déterminants sont extérieurs à l'expérience immédiate du monde et requièrent interprétation. Cela inclut, par exemple, une croyance en une récompense ou une punition qui surviendraient après la mort, déterminées en partie par les circonstances du trépas, mais dont la vérification directe est rigoureusement impossible.

Deuxièmement, c'est également un univers pratique, c'est-à-dire qu'un certain nombre de rituels s'y déploient. Pour plusieurs raisons, ceci est différent d'un terrorisme marxiste ou maoïste, par exemple, puisque ces derniers visent l'établissement d'un monde qui n'existe pas, dont les préceptes ne peuvent être mis en pratique avant la révolution (sauf à une échelle purement locale). Le terroriste religieux, au contraire, vit déjà dans l'univers pratique imposé par son système de croyances. 
Pour les besoins de cette analyse, nous avons identifié et catégorisé les terrorismes possédant ces deux caractéristiques comme «religieux».

Bien sûr, il n'y a pas que le terme de «religieux» qui nécessite d'être précisé: nul besoin de souligner les problèmes de définition du terrorisme (voir le dossier ERTA sur la question à www.erta-tcrg.org). Pour les besoins de cette recherche, le terrorisme comprend tout acte de violence ou de menace de violence coercitive à motif politique visant des non-combattants. Cette définition a l'avantage de cerner la catégorie «terrorisme» à l'aide des tactiques et des méthodes employées et non avec une évaluation subjective de la rationalité, de la fréquence, de la difficulté ou de la moralité des objectifs visés par les individus. Enfin, notons que nous subsumons tout simplement la religion sous la catégorie générale de la politique. Ceci est une simplification très utile et facilement justifiable, surtout lorsqu'il est question des motifs de terroristes religieux, qui confondent eux-mêmes entièrement et délibérément religion et politique. En fait, la distinction souvent faite entre religion et politique est essentiellement moderne et occidentale.

Le matériel empirique utilisé dans l'analyse qui suit provient d'une banque de données constituée par l'Équipe de recherche sur le terrorisme et l'antiterrorisme (ERTA, erta-tcrg.org), du Centre international de criminologie comparée (CICC) de l'Université de Montréal. Fondée en partie sur une étude antérieure (Kellett et al., 1991), elle comprend pour la période 1973-2006 quelque 400 incidents terroristes, dont la plupart sont d'une gravité minime. La première partie de l'exposé consiste à décrire l'état du terrorisme religieux (tel qu'il est défini cidessus) au Canada entre 1973 et 2006. Nous verrons ensuite comment s'articulent les différents rationnels sous-tendant les activités recensées. En conclusion, nous évaluons la pertinence du concept de «nouveau terrorisme» et son utilité à la fois théorique et pratique au regard de la situation au Canada.

\section{Portrait du terrorisme religieux au Canada, 1973-2006}

En général, l'activité terroriste au Canada est particulièrement peu intense et implique surtout de la violence contre des monuments, des édifices et plusieurs types d'installations, qui furent la cible de vandalisme, d'incendies criminels et de quelques rares bombes, pour la plupart de très faible puissance (Leman-Langlois et Ouellet, 2007). Cela 
est vrai non seulement du terrorisme à motif religieux, mais également de l'ensemble de l'activité terroriste au Canada durant la période visée, qu'il soit question de militants pour les droits des animaux, de supporters de groupes néonazis ou d'individus réagissant violemment à diverses étapes de l'évolution de la question de la langue française au Québec. Pour tous les types de terrorisme, les incidents de violence grave sont extrêmement rares.

Dans le cas du terrorisme à motif religieux, les 35 années à l'étude comprennent 116 incidents et peuvent se découper en trois périodes assez distinctes, bien que les séries d'événements qui les caractérisent se chevauchent légèrement. La figure 1 illustre la succession des périodes. Chaque plage temporelle se définit par la dominance d'un certain type de terrorisme, lui-même profondément modifié par son contexte historique.

F I G URE 1

Plages temporelles à l'étude

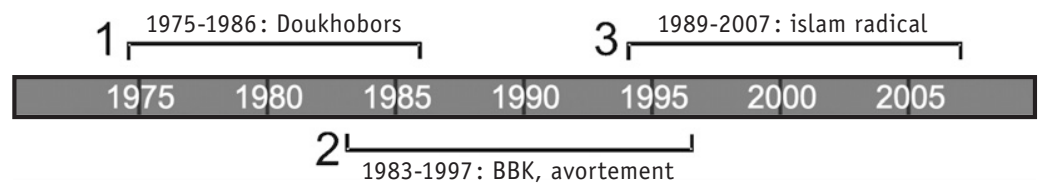

La première s'étend de 1975 à 1986 et comprend 47 attentats commis par des membres de la secte ultraorthodoxe des Doukhobors. Il s'agit, en majeure partie, d'attaques contre des cibles inanimées et surtout des édifices gouvernementaux, des centres communautaires érigés dans leurs villages ou des monuments divers. Les Doukhobors radicaux favorisaient la destruction par le feu, réduisant en cendres sept maisons, trois salles communautaires, un entrepôt coopératif, un entrepôt appartenant au Canadien Pacifique et quatre musées historiques. Quelques bombes furent également placées sur deux pylônes de ligne à haute tension, un pylône de téléphone et sur trois tronçons de voies ferrées du Canadien Pacifique. S'ajoutent à ces cas plusieurs tentatives ratées d'incendier ou de faire exploser d'autres cibles du même genre et des cas de monuments détruits ou vandalisés.

La seconde période à l'étude couvre les années 1983-1997 (17 incidents) et comporte deux séries distinctes d'incidents. La première série est celle des activités d'extrémistes sikhs dans l'Ouest canadien qui, 
contrairement à l'histoire des Doukhobors, aboutirent à un acte terroriste de proportions historiques (le deuxième plus meurtrier après l'attentat de 2001 contre le World Trade Center). Les activités des membres du groupe Babbar Khalsa (BK et BKI, pour Babbar Khalsa International) commencent en 1978 avec le meurtre d'une des têtes dirigeantes du groupe, torturé et pendu, à la suite d'une lutte de pouvoir interne en Colombie-Britannique. Un autre conflit du genre éclate en 1982, à Toronto cette fois, et un membre d'une des factions en opposition décharge une arme en plein tribunal, tuant deux personnes et blessant une troisième. Toujours en 1982, une émeute devant l'ambassade indienne fit un blessé lorsqu'un participant fit feu dans la foule. La violence culmine en 1984 et 1985, à la suite du massacre de séparatistes sikhs en Inde. Le 23 juin 1985, une bombe fait s'écraser dans l'Atlantique le vol 182 d'Air India, tuant 329 personnes. Une seconde bombe, heureusement mal conçue, explose lors d'un transfert de bagages à l'aéroport Tokyo/Narita, tuant deux employés. En 1986, un ministre indien en visite est attaqué à l'arme à feu, mais survit à ses blessures. Enfin, en mai de la même année, cinq Sikhs de Montréal sont arrêtés par la GRC pour un complot qui visait à répéter l'exploit du vol 182.

Nous trouvons également dans la période 1983-1997 une dizaine d'incidents causés par des opposants à l'accès libre à l'avortement, tous d'inspiration fondamentaliste chrétienne. Le docteur Morgentaler est victime de plusieurs tentatives de voies de fait, dont une où l'agresseur présumé, maîtrisé par la foule, brandissait des sécateurs de jardinage. Les cliniques du docteur sont également ciblées, attaquées à la bombe, à l'acide, aux outils lourds, et incendiées. Dans les années 1990, trois médecins sont victimes de tentatives de meurtre alors que James Kopp parcourt le pays dans la mission autoattribuée de dissuader les médecins d'offrir les interventions.

La troisième période s'étend entre 1989 et 2007 (38 incidents), avec une augmentation marquée entre 2001 et 2007 (26 incidents, 68\%). Il s'agit d'activités d'extrémistes s'identifiant explicitement ou implicitement à l'Islam. Dès les années 1980, des extrémistes islamistes se lançaient dans une campagne d'intimidation, à la suite de la publication des Versets sataniques de Salman Rushdie. Plusieurs commerces offrant l'ouvrage furent vandalisés et des ministres du cabinet conservateur de l'époque (Otto Jelinek, Revenu; Joe Clark, Affaires étrangères) reçurent des menaces de mort. En 1995, une bombe de $250 \mathrm{~kg}$ de dynamite rase complètement l'ambassade d'Égypte au Pakistan. Une des têtes 
dirigeantes de l'attentat était Ahmad Saeed Khadr, Égyptien naturalisé Canadien. Arrêté, Khadr se lança dans une grève de la faim pour clamer son innocence. Le premier ministre canadien d'alors, Jean Chrétien, intercéda en sa faveur auprès de son homologue pakistanaise, Benazir Bhutto. Khadr fut éventuellement relâché, après quoi il rejoint le Front islamique international d'Oussama ben Laden («al Qaïda») avec sa famille.

Durant la fin des années 1990, plusieurs terroristes islamistes sont arrêtés alors qu'ils tentent de traverser la frontière entre le Canada et les États-Unis. Hani Al Sayegh, par exemple, est saisi par la GRC après avoir réussi à échapper à l'Immigration and Naturalisation Service (INS) étatsunien. Al Sayegh avait pourtant participé, en 1996, à la destruction des tours Khobar (Arabie Saoudite), qui avait tué 19 soldats étatsuniens. Le cas le plus notoire se produisit en sens inverse, alors qu'Ahmed Ressam tenta de traverser la frontière en transportant dans le coffre de sa voiture une bombe artisanale qu'il avait lui-même mise au point (avec l'aide de trois complices). Ayant contracté la malaria, fiévreux, Ressam répondit de manière désorganisée aux questions d'une douanière étatsunienne, qui décida de fouiller son véhicule. Il avoua à son procès avoir eu pour objectif de s'attaquer à l'aéroport de Los Angeles.

Depuis 2001, les activités d'extrémistes musulmans canadiens ici et ailleurs dans le monde se sont multipliées. Par exemple, en Afghanistan, les fils de Ahmad Khadr sont arrêtés un à un alors qu'ils résistent aux troupes de la coalition. Khadr père est lui-même tué; un de ses fils est paraplégique, un autre attend son procès à Guantanamo. Plus près de nous, notons des cas d'esprits échauffés qui ont incendié des écoles juives (Talmud-Torah, le 5 avril 2004 et Skver-Toldos le 5 septembre 2006) au nom de groupes ou de personnes associés au jihadisme international.

Nous comptons également cinq cas de complots terroristes. Le premier fut mis au grand jour par l'arrestation, à Burnaby (C.-B.), d'Ali Adham Amhaz, impliqué dans la fourniture de matériel (Tazers, lunettes de vision de nuit, dispositifs GPS, etc.) et de fonds au profit du Hezbollah. Deuxièmement, Mohammed Jabarah, citoyen canadien arrêté en mars 2002, fut lié à des enregistrements vidéo trouvés dans des ruines d'édifices occupés par des jihadistes en Afghanistan. Troisièmement, en 2003, un appel de menaces est jugé suffisamment sérieux pour que les vols de la compagnie israélienne El Al en direction de Toronto soient temporairement déviés vers Hamilton. Il semble que 
les avions aient été potentiellement visés par un tir de missile sol-air aux environs de l'aéroport Pearson. Quatrièmement, le 30 mars 2004, Mohammed Khawaja, résidant d'Orleans dans la banlieue d'Ottawa, est arrêté pour avoir porté assistance à un groupe d'extrémistes londoniens (liés au groupe ayant commis les attentats meurtriers du 7 juillet 2005 - la plupart furent condamnés à perpétuité en mai 2007). Fait intéressant, c'est l'interception de ses courriels par la National Security Administration (NSA) étatsunienne qui identifia Khawaja comme conspirateur. Il y expliquait comment il avait mis au point un détonateur électronique particulièrement efficace. Il devint ainsi le premier individu arrêté sous le coup des nouvelles dispositions du Code criminel instaurées par la Loi antiterroriste de 2001 et criminalisant le soutien à des activités terroristes. Enfin, impossible d'ignorer le cas du «complot de Toronto», qui mena à l'arrestation de 18 hommes en juin et août 2006 (à quoi il faut additionner deux citoyens étatsuniens arrêtés en 2005). Clavardant sous surveillance, allant s'entraîner au jihad dans le Nord ontarien (sous supervision d'un agent infiltré du Service canadien de renseignement de sécurité, SCRS) et se procurant (auprès d'un agent de la GRC) quelque trois tonnes de nitrate d'ammonium, il faudra attendre la fin de leur procès pour comparer l'intensité de leur enthousiasme à celui des multiples agents de police qui les ont assistés. Au pire, le groupe préparait des explosions majeures au pied de la tour du $\mathrm{CN}$ et devant les bureaux du SCRS sur la rue Front, peut-être accompagnées d'une attaque contre le Parlement, durant laquelle le premier ministre Stephen Harper devait avoir la tête tranchée. Le passage de cette exubérance irréfléchie - mais somme toute parfaitement typique du clavardage - à l'acte plus concret de se procurer un ingrédient d'une éventuelle bombe a sans aucun doute grandement bénéficié du catalyseur policier.

Avant de clore cette section descriptive, il est utile de souligner que l'activité des gouvernements et des forces de l'ordre, surtout durant cette période, fut infiniment plus intense que celle des individus décrits cidessus. Les cas d'arrestations préventives, de détention sous les fameux "certificats de sécurité», les nouveaux règlements et interdictions, le déblocage de nouveaux fonds publics, la création de nouvelles agences, etc. sont beaucoup plus nombreux et feront l'objet d'un article subséquent. Nos statistiques étant à la remorque de ces activités, un certain effet d'augmentation est certainement dû à l'intensité des activités policières, surtout dans le cas des complots déjoués. 


\section{La rationalité du terrorisme religieux au Canada}

Comme le montrent les chiffres ci-dessus, les incidents de terrorisme religieux au Canada sont, somme toute, extrêmement rares. Il est donc impossible de procéder à des analyses statistiques qui décèleraient une tendance ou qui permettraient des prédictions à court ou à long terme. Néanmoins, nous pouvons explorer et tirer certaines caractéristiques généralisables au sujet des rationalités terroristes religieuses. Dans la présente section, nous proposons une catégorisation en trois principaux types.

Terroristes à objectif spécifique défini par leur religion

Le terroriste à objectif spécifique est engagé dans un combat violent contre une pratique ou une politique qu'il juge contraire à un quelconque principe moral défini par sa religion. Au Canada, il s'agit surtout de groupes ou d'individus détruisant des installations et du matériel, menaçant et agressant des personnes afin de mettre fin à l'avortement sur demande. Ce type d'activité a donné lieu à plusieurs attentats au Canada, dont trois attentats par arme à feu contre des obstétriciensgynécologues par James Kopp, qui continue d'affirmer que ses actes sont moralement justifiés (il est présentement incarcéré à perpétuité pour le meurtre d'un autre médecin aux États-Unis). Plusieurs groupes et individus offrent un soutien moral et financier à Kopp par l'entremise de leurs sites Internet (dont Army of God et Christian Gallery).

\section{Terroristes imposant leur religion}

Ces terroristes sont engagés dans des activités de contrôle social, généralement au niveau local. Leurs actions visent à forcer les individus qu'ils identifient comme appartenant à leur groupe religieux à respecter les dogmes, pratiques, rituels, etc. tels qu'ils les comprennent, accusant souvent leurs coreligionnaires de dissolution ou d'impiété.

Les Doukhobors, après leur arrivée de Russie à la fin du XIx siècle, se sont scindés en plusieurs sous-groupes, dont un très fondamentaliste nommé «Fils de la liberté». Ces derniers sont responsables de la plupart des actes terroristes décrits ci-dessus. Les deux principaux motifs de leurs attaques étaient, d'une part, leur perception d'une trop grande incursion du monde extérieur aux abords et à l'intérieur de leurs com- 
munautés et, d'autre part, le constat que de plus en plus de Doukhobors violaient les règles interdisant la propriété privée, l'utilisation d'objets technologiquement avancés ou l'idolâtrie. Ainsi, la plupart de leurs actes de destruction et de vandalisme visaient à faire disparaître ou à rendre inutilisables les objets ou édifices jugés impies, comme les musées, ou ceux qui représentaient le monde extérieur.

Terroristes à objectifs larges structurés par un discours religieux

Ici, le terroriste vise des objectifs très larges, voire historiques, définis à l'aide de sa religion. Notons que pour certains terroristes, la religion sert davantage de mode d'identification des groupes et des individus impliqués. Par exemple, les membres de plusieurs groupes se reconnaissent par leur appartenance religieuse, comme c'est le cas du terrorisme catholique ou protestant en Irlande. D'autres groupes se reconnaissent par leur identification d'un ennemi amalgamé à une vision particulière de sa religion. C'est le mode de fonctionnement de plusieurs groupes antisémites. Suivant notre définition, nous ne considérons pas ces deux cas de figure comme du "terrorisme religieux» puisque l'élément central du motif n'est pas religieux.

L'exemple de l'extrémisme sikh a ceci de particulier de s'être fortement ancré dans des croyances religieuses à partir du milieu des années 1980, essentiellement en réaction à l'attaque contre le complexe sikh du Temple d'or, à Amritsar. À ses débuts, le nationalisme séparatiste sikh visait explicitement la création d'un pays indépendant, fondé surtout sur le différentiel culturel des Sikhs, dont la religion était bien sûr un ingrédient déterminant (d'ailleurs, le nom de ce pays éventuel, «Khalistan», signifie «pays des purs»). Le dixième gourou du sikhisme et fondateur du Khalsa, Gobind Singh Ji, est réputé avoir placé l'indépendance comme condition de survie de la religion sikhe. Néanmoins, à cette époque, le discours restait centré sur les revendications territoriales.

En 1966, une première tentative de dissoudre le mouvement séparatiste consista à créer la province du Penjab, dont les frontières correspondaient à peu près aux terres occupées par les Sikhs - tout en incluant une grande proportion de non-sikhs peu sympathiques à l'indépendance. Au début des années 1980, le gouvernement d'Indira Ghandi avait opté pour une stratégie politique plus risquée: financer un nouveau parti indépendantiste pour diviser l'opposition. Ayant perdu 
le contrôle de ce nouveau groupe, de plus en plus militant, l'armée indienne dut maîtriser une quasi-rébellion, en juin 1984, en investissant et neutralisant plusieurs positions retranchées des insurgés. L'opération, nommée «Bluestar», fut un désastre monumental. L'une des zones barricadées était le complexe politico-religieux d'Amritsar. Durant l'assaut, qui fit plus de 500 morts, l'Akal Takht, siège du l'autorité sikhe, fut fortement endommagé. Plusieurs autres édifices furent également bombardés ou incendiés, laissant une trace symbolique importante.

Au Canada, ceci marqua une radicalisation sensible du discours séparatiste, d'ailleurs notée par les services de renseignements (Bolan, 2005). Auparavant surtout axées sur la collecte de fonds, les organisations séparatistes sikhes devinrent de plus en plus radicales.

Davantage d'actualité, le discours terroriste jihadiste d'extrémistes musulmans apparaît à l'extrême de ce type de terrorisme (bien que leurs actes restent pour l'instant d'une gravité de beaucoup inférieure). On peut y trouver quelques objectifs relativement circonscrits, comme le retrait des troupes infidèles des terres sacrées d'Arabie Saoudite et d'ailleurs, mais le noyau conceptuel autour duquel s'articule l'ensemble est vaste, grandiose et implique que des changements radicaux s'opèrent à l'échelle du globe, au niveau économique, géopolitique et bien sûr religieux. En un mot, l'objectif ultime est le retour du Califat, âge d'or du monde islamique, époque idéalisée et largement mythique, durant laquelle la domination de la culture musulmane s'étendait sur une grande partie de l'Europe, de l'Asie et de l'Afrique. Ainsi, la mondialisation à laquelle l'extrémiste musulman s'oppose n'est pas le concept même d'une internationalisation de la culture ou des échanges commerciaux et autres, contrairement à ce qu'affirment plusieurs auteurs (dont Stern, 2003), mais bien la mondialisation occidentale, qui réduit toujours davantage la place comprise comme revenant de droit au monde musulman.

Que l'adhérent individuel réalise ou non la démesure et l'improbabilité pratique de cet objectif ultime est sans importance. D'une part, un certain nombre d'objectifs immédiats ou intermédiaires, censés mener au but final, s'offrent à lui. D'autre part, bien qu'il comprenne sans doute que les chances de réussite totale sont faibles, sinon nulles, il s'agit d'un combat de la vertu seule. Mourir en essayant est donc aussi valable que de réussir.

Paradoxalement, cela peut mener à une lutte d'apparence purement ritualiste, que certains ont qualifiée de nihiliste, ce qui est profondément erroné. Le fait que des objectifs clairs et rationnels ne soient pas expli- 
citement articulés ne doit pas être confondu avec l'absence d'objectifs. Le fait que les moyens d'atteindre ces objectifs nous paraissent démesurés, immoraux ou irrationnels non plus. Notons d'ailleurs que le passage à un certain ritualisme n'est pas l'apanage des jihadistes. Torrance (1986) a remarqué que les Doukhobors, à l'époque la plus intensive de leur lutte (1950-1960), perpétrèrent un grand nombre d'attaques indiscriminées, comme si la rationalité instrumentale liée à la construction de bombes et à leur installation avait temporairement pris le dessus. Quoi qu'il en soit, cette dérive ritualiste est très rare. Il faut également tenir compte du fait qu'un groupe n'est jamais qu'un ensemble d'individus, dont les buts particuliers, ainsi que la compréhension personnelle des buts du groupe, diffèrent. Ceci peut facilement mener à l'apparence d'irrationalité chez le groupe, mais cette apparence est le résultat d'une erreur de conceptualisation de la part de l'observateur, habitué à traiter les groupes comme des entités pensantes homogènes et durables.

En terminant cette section, un certain nombre d'observations s'imposent. Premièrement, aucun de ces terroristes ne cherche le soutien d'une partie du public. Le terrorisme politique traditionnel était hautement dépendant de l'acceptation de ses objectifs, voire de ses moyens, par une «masse critique» de concitoyens, sur laquelle il dépendait pour se cacher, pour faire pression sur le gouvernement, pour lui apporter un soutien financier et logistique, etc. Un des facteurs de la disparition du Front de libération du Québec, par exemple, fut justement la réaction négative de la population à la mort d'un des otages capturés (LemanLanglois et Brodeur, 2005). Au contraire, le message du terroriste religieux ne vise pas à gagner de nouveaux fidèles ou à mobiliser la population. Cela a pour conséquence principale que les moyens adoptés par les terroristes ne sont plus limités par ce qu'ils croient que le citoyen moyen peut accepter.

Deuxièmement, alors que la pensée dominante de la plupart des groupes terroristes conventionnels présente l'action militante comme une suite d'étapes vers l'accomplissement d'un idéal futur, les terrorismes religieux sont surtout orientés vers le passé. Les extrémistes sikhs tentent de se venger de l'Inde, les extrémistes musulmans tentent de redonner vie à une époque révolue, les fondamentalistes Doukhobors, en plus d'idéaliser un monde épuré de toute technologie, concevaient aussi toute adaptation de leurs coreligionnaires à la modernité comme un égarement nécessitant un retour de force à la tradition. 
Troisièmement, aucun des terroristes décrit dans les exemples cidessus n'a laissé de revendications ou d'autre forme de message explicite pour justifier ses actes ou pour exiger une chose ou une autre. Dans la plupart des cas, le message est implicite, contenu dans l'acte même, ou dans une affiliation déclarée. Il est logique de supposer, par exemple, que ceux qui se réclament du Front islamique international ou d'al Qaïda en partagent les idéaux, du moins dans les grandes lignes. Non pas que ben Laden ou ses acolytes aient accompagné leurs actes de messages plus clairs: aucun message spécifique n'a suivi les attentats contre les ambassades étatsuniennes au Kenya ou en Tanzanie, l'attaque du USS Cole ou celle du 11 septembre. Cependant, ben Laden et en particulier Ayman al Zawahiri, autre tête du réseau, ont chacun produit un grand nombre de déclarations «officielles» sur une foule d'aspects du monde contemporain et du jihad (pour un recueil assez complet, voir Lawrence, 2005), qui ont été largement diffusées dans les médias. Il serait exagéré de supposer que le moindre jihadiste soit parfaitement au courant de l'ensemble de ces discours, et encore moins qu'il les comprend tous, mais l'important est que la supposition que la «Vérité» est disponible et a été dite remplace le besoin individuel de s'exprimer.

Enfin, notons que seule notre troisième catégorie de terrorisme religieux peut être apparentée à ce que nous avons brièvement décrit comme le «nouveau terrorisme» annoncé par plusieurs auteurs. Chronologiquement, comme il a été noté dans notre deuxième section, c'est aussi celui qui est présent de nos jours, les deux autres ayant pour l'essentiel disparu.

\section{Nouveau terrorisme et justification par l'absolu}

Parler de la rationalité du terroriste religieux implique une définition ni aristotélicienne, ni économique, mais weberienne du concept de rationalité. C'est une rationalité au sens où l'action est le résultat d'une réflexion, d'une certaine logique; c'est toutefois une logique strictement interne, individuelle, et qui peut paraître illogique ou «irrationnelle» à l'observateur - d'où l'enlisement de plusieurs recherches dans les tentatives infructueuses d'expliquer le terrorisme comme une pathologie mentale (Mannoni, 2004). Qu'on se penche sur Ahmed Ressam ou sur Omar Bulphred (illustre inconnu qui entreprit en 2006-2007 une campagne de bombes à faible puissance contre des écoles et centres 
communautaires juifs à Montréal), tenter de comprendre leurs actes à l'aide de la grille de l'analyse coûts/bénéfices est peu productif.

C'est plutôt une rationalité normative qui prime, ce qui est d'ailleurs le propre de la plupart des formes de pensée religieuse (Jerolmack et Porpora, 2004). Cette nuance importante force Pape (2005), qui évacue le facteur religieux entièrement, à introduire à sa place la notion de «stratégie altruiste» pour contourner le problème conceptuel d'un suicide produisant davantage de bénéfices que de coûts. Cela reste insuffisant. S'il faut tirer un enseignement des faits exposés ci-dessus, c'est que cette dichotomie rationnel-irrationnel est trompeuse, qu'on l'utilise pour faire de la rhétorique, de la science ou de la philosophie. Dans les faits, un ensemble de rationalités normatives animent aussi bien les dirigeants et les exécutants que les terroristes qui agissent entièrement seuls. Ils passent à l'action non pas parce que des bénéfices éventuels sont perceptibles, ou à l'intérieur d'une stratégie altruiste, mais parce qu'ils ressentent une injustice, parce qu'ils sont mis en colère par une humiliation perçue, ou parce qu'ils sont convaincus qu'il n'en tient qu'à eux pour que la volonté de Dieu soit faite. Autrement dit, le terrorisme est un acte moral, le terrorisme religieux un acte moral religieux.

Non pas que le terroriste mû par la religion évolue dans un univers strictement surnaturel, évidemment. Le monde du terroriste est également formé d'événements politiques, de luttes armées, de catastrophes humanitaires, de répression, de pauvreté, etc. - tout comme le nôtre. La différence réside dans l'interprétation qui est faite de ce monde et dans le potentiel d'engagement de l'observateur. Dans les deux cas, la présence d'absolus est particulièrement déterminante. D'une part, les vérités absolues révélées par la religion ne sont pas du même ordre que les vérités scientifiques, culturelles, traditionnelles qui sont offertes par l'activité humaine. Ainsi, toute nouvelle information doit être filtrée et interprétée pour se conformer aux vérités absolues déjà connues. C'est d'ailleurs un exercice auquel se livrent systématiquement un grand nombre de théologiens de toutes les religions. D'autre part, la mobilisation de l'individu est également favorisée par la présence d'absolus normatifs qui définissent l'action et l'inaction, les groupes d'acteurs, l'ordre, les règles etc. en termes manichéens.

Cela explique qu'une large portion de ce qui a été nommé (trompeusement) terrorisme «maison» (bomegrown terrorism), qui est le fait d'enfants d'immigrants, généralement musulmans (ou convertis), soit 
excessivement peu ou mal organisé. Ce terrorisme, spontanément déclenché par des événements, est d'un amateurisme souvent désarmant. Le complot de Toronto l'illustre: en discutant de leur attaque sur Internet, les membres du groupe dissimulèrent leur identité par la simple adoption d'un pseudonyme, stratagème notoirement inadéquat. L'un d'eux fut facilement mis sous surveillance lorsque les autorités s'aperçurent qu'il parasitait les réseaux sans fil à proximité de son lieu de résidence - les policiers s'empressèrent de lui en offrir un, occasion qu'il saisit aussitôt. Un autre était déjà connu de la communauté pour avoir souvent tenu en public un discours particulièrement belliqueux - sa colère à l'endroit des fléaux affligeant l'Umma ayant de beaucoup précédé sa décision de poser des bombes. Cette autoidentification en tant que radical est bien sûr peu recommandée pour ceux qui veulent s'adonner à des activités clandestines.

Le lecteur aura noté que les terroristes religieux responsables des actes les plus graves au Canada sont musulmans et sikhs. Inutile de discuter de la rectitude de l'interprétation qu'ils font de divers aspects des édits des dix gourous, du Coran, des hadiths ou de la charia. C'est là une question théologique sans importance ici, comme celle de savoir si le Coran est effectivement la parole de Dieu ou s'il est «rationnel» de croire à des puissances surnaturelles. La question plus sociologique de la correspondance de cette interprétation avec celle de la majorité de leurs coreligionnaires (une intersubjectivité religieuse) est plus intéressante, mais semble avoir un impact limité. D'une part, même si la plupart des musulmans rejetaient les interprétations belliqueuses de leur tradition religieuse, la proportion infime de ceux qui les adoptent représentera toujours un nombre suffisamment important pour que des actes violents soient possibles. Aucune théologie ne sera jamais suffisamment hégémonique pour éliminer entièrement les dissensions. D'autre part, les «purs» ne sont aucunement perturbés par le fait qu'un grand nombre de leurs coreligionnaires les considèrent comme des extrémistes dangereux. Les Sikhs extrémistes, d'ailleurs, choisirent tout simplement d'éliminer un éditeur qui s'opposait à leur discours revanchard et fondamentaliste (Tara Singh Hayer, paralysé par balle en 1988 et tué en 1998).

Ici, Sellin (1938) reste d'actualité et peut nous être d'une aide précieuse. Dans son étude des activités délinquantes des membres de minorités immigrantes, il avait distingué les générations successives d'immigrants selon leur mode d'intégration à leur société d'accueil. Si les premiers arrivants étaient à l'occasion en position de «conflit cultu- 
rel» lorsque certaines de leurs pratiques traditionnellement acceptées étaient interdites par les codes locaux, leurs enfants vivaient plutôt une forme de conflit interne, de contradiction dans leur propre échelle de valeurs. Produits du frottement des cultures et du relativisme immédiat, irréfléchi qui en découle, les enfants de seconde et de troisième génération étaient plus facilement enclins à la déviance que la moyenne. En fait, une forme de tension émergeait de la difficulté, pour l'adolescent, de s'intégrer à la fois à la culture dominante et à celle de ses parents. Cette tension donnait lieu à divers modes d'adaptation, dont en particulier une forme rare mais intense de «retrait» où l'individu en venait à glorifier sa représentation de sa culture «originelle» et à mépriser la culture de son pays d'accueil. On voit facilement à quel point ces observations s'appliquent au terrorisme des enfants et des petits-enfants d'immigrants (l'appellation de «terrorisme maison» provient de l'idée, erronée, qu'à la base tout terrorisme est nécessairement international, c'est-à-dire venu d'ailleurs).

Ainsi, dans les pays occidentaux le facteur déclencheur du terrorisme religieux est la combinaison des conditions d'intégration des immigrants et de leurs descendants et de l'adoption d'une rationalité normative absolue visant au départ à réduire un problème d'identité culturelle mais se développant, au gré des événements internationaux, en un désir de passer aux actes. Le facteur catalyseur est sans aucun doute l'élément principal du modèle réseauté de Sageman (2004), c'est à dire la rencontre et l'interaction avec d'autres individus pareillement disposés (Sageman reste toutefois muet sur les conditions initiales d'entrée dans un réseau). Sauf rares exceptions, le terrorisme religieux, comme le terrorisme en général, est une activité de groupe.

\section{Conclusion : religion et rationalité terroriste}

Au Canada comme ailleurs, la rationalité normative du terroriste religieux se déploie autour de la création d'une identité personnelle et sociale particulière aux tensions issues de l'adaptation des immigrants et de leurs enfants à leur société d'accueil. Cette rationalité est absolue, manichéenne, implique des objectifs grandioses, historiques, épiques - dont la réalisation est donc improbable - et la disparition de la plupart des limites applicables aux tactiques adoptées. Il implique également un discours légitimant reposant en tout ou en partie sur des référents tenant 
à la foi, c'est-à-dire radicalement invérifiables et incompatibles avec tout compromis ou négociation.

Cet article ne s'est pas penché en profondeur sur les motifs particuliers de chaque terroriste étudié, mais plutôt sur la rationalité générale dans laquelle les motifs s'imbriquent. C'est qu'en eux-mêmes les motifs sont bien peu: qu'on désire venger telle ou telle injustice, restaurer tel ou tel état de fait, la puissance mobilisatrice réside dans le discours général qui donne lieu aux motifs. Il serait erroné de supposer que les agents décrits dans ce qui précède sont des individus mobilisés par l'adoption d'objectifs particuliers (comme le voleur est mobilisé par le désir de s'approprier un objet). Comme le montrait déjà C. Wright Mills (1940), il est plus productif de comprendre les motifs comme des outils conceptuels permettant à l'individu d'ordonner le monde et d'identifier des problèmes et des solutions. En passant des motifs (pourquoi j'ai agi ainsi) aux valeurs (pourquoi doit-on agir ainsi, en général) le rationnel religieux est également un moyen de s'adresser aux autres, d'exercer un pouvoir sur eux, de passer de l'action individuelle à l'action de groupe.

Le terrorisme religieux canadien n'est pas un produit direct des conditions sociopolitiques qu'on a souvent accusées d'être la source de terrorisme dans des pays du tiers monde. Il est plutôt le produit d'une identification à ceux qui les vivent. Ainsi, il reste essentiellement impossible à prévoir, puisque sujet aux aléas des événements qui font irruption sur la scène internationale. Par contre, puisque ce sont ceux qui sont déjà les plus impliqués dans un rationnel, dans un discours extrémiste, donc ceux qui se sont autoidentifiés, qui risquent le plus de passer aux actes, une certaine surveillance préventive reste envisageable - bien que le moindre abus de cette surveillance sera certainement identifié comme un élément de corroboration du rationnel de victimisation religieuse et ainsi risquera de faire partie des événements déclencheurs.

\section{Références}

Bolan, K. (2005). Loss of Faith: How the Air India Bombers Got Away With Murder. Toronto: McClelland \& Stewart.

Campbell, J. (1999). La secte japonaise Aum Shinrikyo. In G. Chaliand, Les stratégies du terrorisme (163-193). Paris: Desclée de Brouwer.

Chidester, D. (1988). Rituals of Exclusion and the Jonestown Dead. Journal of the American Academy of Religion, 56 (4), 681-702. 
Davis, W. (2000). Heaven's Gate: A Study of Religious Obedience. Nova Religio, 3 (2), 241-267.

Dein, S., \& Littlewood, R. (2000). Apocalyptic suicide. Mental Health, Religion $\mathfrak{}$ Culture, 3 (2), 109-114.

Duyvesteyn, I. (2004). How New Is the New Terrorism? Studies in Conflict 6 Terrorism, 27 (5), 439-454.

Gusfield, J. (1986). Symbolic Crusade: Status Politics and the American Temperance Movement. Chicago: University of Illinois Press.

Hall, J. (1995). Public Narratives and the Apocalyptic Sect: From Jonestown to Mt. Carmel. In S. Wright (ed.), Armageddon in Waco: Critical Perspectives on the Branch Davidian Conflict (205-235). Chicago: University of Chicago Press.

Heaton, P. (2006). Does Religion Really Reduce Crime? Journal of Law and Economics, XLIX, 147-172.

Hirschi, T., \& Stark, R. (1969). Hellfire and Delinquency. Social Problems, 17, 202-213.

Introvigne, M. (1995). Ordeal by Fire: The Tragedy of the Solar Temple. Religion, 25 (3), 267-283.

Introvigne, M., \& Mayer, J.-F. (2002). Occult Masters and the Temple of Doom: The Fiery End of the Solar Temple. In D. Bromley \& J. Melton (eds.), Cults, Religion and Violence (170-188). Cambridge: Cambridge University Press.

Jerolmack, C., Poropra C., \& Poropra D. (2004). Religion, Rationality and Experience: A Response to the New Rational Choice Theory of Religion. Sociological Theory, 22 (1), 140-160.

Johnson, B., Jang, S. J., Larson. D., \& De Li, S. (2001). Does Adolescent Religious Commitment Matter? A Reexamination of the Effects of Religiosity on Delinquency. Journal of Research in Crime and Delinquency, 38 (1), 22-44.

Kellett, A., Beanlands, B., Deacon, J., Jeffrey, H., \& Lapalme, Ch. (1991). Terrorism in Canada, 1960-1989. Ottawa: Ministry of the Solicitor General of Canada, National Security Coordination Centre, Police and Security Branch.

Laqueur, W. (2000). The New Terrorism: Fanaticism and the Arms of Mass Destruction. Oxford: Oxford University Press.

Lawrence, B. (2005). Messages to the World: The Statements of Osama bin Laden. Londres: Verso.

Leman-Langlois, S., \& Brodeur, J.-P. (2005). Terrorism Old and New : Counterterrorism in Canada. Police Practice and Research, 6 (2), 121-140.

Leman-Langlois, S., \& Ouellet, G. (2007). Évolution cyclique du terrorisme politique au Canada, 1973-2005. Présentation au $75^{\mathrm{e}}$ congrès de l'Association francophone pour le savoir (ACFAS), 7-11 mai 2007. Trois-Rivières: ACFAS.

Lifton, R. (2000). Destroying the World to Save It: Aum Shinrikyo, Apocalyptic Violence and the New Global Terrorism. New York: Henry Holt.

Mannoni, P. (2004). Les logiques du terrorisme. Paris: In Press. 
Metraux, D. (1995). Religious Terrorism in Japan: The Fatal Appeal of Aum Shinrikyo. Asian Survey, 35 (12), 1140-1154.

Mills, C. W. (1940). Situated Actions and Vocabularies of Motives. American Sociological Review, 6, 904-913.

Noble, K. (1998). Tabernacle of Hate: Why They Bombed Oklaboma City. Prescott (Ontario): Voyageur Publishing.

Pape, R. (2005). Dying to Win: The Logic of Suicide Terrorism. New York: Random House.

Rand/MITP (2007). MITP Terrorism Knowledge Base, <http://www.tkb. $\operatorname{org} />$.

Rappoport, D. (1984). Fear and Trembling: Terrorism in Three religious Traditions. American Political Science Review, 78 (3), 668-672.

- (1990). Sacred Terror: A Contemporary Example from Islam. In W. Reich (ed.), Origins of Terrorism: Psychologies, Ideologies, Theologies States of Mind (1998, 103-130). Washington (D.C.): Woodrow Wilson Center Press.

Sageman, M. (2004). Understanding Terror Networks. Philadelphie: University of Pennsylvania Press.

Sellin, T. (1938). Conflits de culture et criminalité. Paris: Pedone, 1984.

Sprinzak, E. (1998). The Great Superterrorism Scare. Foreign Policy, Fall 1998, $110-24$.

Stern, J. (2003). Terror in the Name of God: Why Religious Militants Kill. New York: Harper Collins.

- (1999). The Ultimate Terrorists. Cambridge: Harvard University Press.

Torrance, J. (1986). Public Violence in Canada. Montréal: McGill-Queen's University Press.

Urban, H. (2000). The Devil at Heaven's Gate: Rethinking the Study of Religion in the Age of Cyber-Space. Nova Religio, 3 (2), 268-302.

Wilkinson, P. (2006). Terrorism Versus Democracy: The Liberal State Response, (2nd Edition). New York: Routledge.

ABSTRACT - Since the 1990s many analysts of terrorism have adopted the concept and the phrase "new terrorism" in order to account for what they have seen as the emergence of a different form of political violence. New terrorism has been characterised as escaping the boundaries of states and routinely targeting installations and persons for their symbolic content. It is motivated by glorious, if not grandiloquent objectives, with strong supernatural and mythical aspects. Its demands are often impossible to meet since they involve planet-wide reorganisation. Most importantly, it is a religious terrorism, built on absolutes and capable of justifying the most extreme forms of violence - which conventional terrorism tended to avoid. For obvious theoretical, as well as practical reasons, we will try to describe the manifestation of such terrorism in Canada.

Our study rests on a 400 incident qualitative database gathered from Canadian media reports. Our observation period ranges from 1973 to 2006, but the bulk of religious (and other) terrorist activities took place in the 1980s. We then try to account 
for this differential level of activity with the help of a few theoretical tools, in particular through the "social control" function of religious practice. We conclude with an evaluation of the usefulness and explanatory power of the concept of new terrorism.

RESUMEN - A partir de mediados de los años noventa, varios autores han adoptado el concepto de "nuevo terrorismo" para referirse a lo que perciben como una nueva forma de violencia política. Un tipo de terrorismo que rebasa el ámbito de los Estados y selecciona blancos según su contenido simbólico, con objetivos gloriosos y múltiples elementos sobrenaturales o legendarios, lo que conduce a pocas reivindicaciones concretas o realizables. Es también - y sobre todo - un terrorismo de gran contenido religioso, absolutista, que va acompañado de una violencia que desconoce los límites establecidos por el terrorismo convencional. Por razones a la vez teóricas y prácticas, es importante comprender cómo y hasta qué punto este nuevo terrorismo se manifiesta en Canadá.

A partir de una base de datos cualitativa de casi 400 incidentes y situaciones de violencia política acaecidos en Canadá desde 1973, este artículo establece ciertos hechos, entre ellos el escaso número de atentados religiosos y su casi desaparición a partir de los años ochenta. Asimismo, se analiza esta evolución y se ofrecen algunas explicaciones, entre ellas la función del control social, a menudo atribuido a la práctica religiosa. Finalmente, se revisa la pertinencia del concepto "nuevo terrorismo" y su utilidad tanto teórica como práctica. 